\title{
Matéria orgânica e valor nutricional do solo na produção de pastagens irrigada na
}

\section{Caatinga Piauiense}

Organic matter and soil nutritional value in the production of irrigated pastures in the Caatinga

Piauiense

Materia orgánica y valor nutricional del suelo en la producción de pastos de regadío en la Caatinga

Piauiense

Recebido: 19/10/2021 | Revisado: 28/10/2021 | Aceito: 04/11/2021 | Publicado: 08/11/2021

Adriano Veniciús Santana Gualberto
ORCID: https://orcid.org/0000-0002-1764-5564
Universidade Federal do Piauí, Brasil
E-mail: a.vsg @ hotmail.com
João Rodrigues da Cunha
ORCID: https://orcid.org/0000-0001-6990-3361
Universidade Federal do Piauí, Brasil
E-mail: joaorcsolos@ hotmail.com
Renato Falconeres Vogado
ORCID: https://orcid.org/0000-0003-3282-7363
Universidade Federal da Paraíba, Brasil
E-mail: renatoagro86@ @otmail.com
Paloma Cunha Saraiva
Paloma
ORCID: https://orcid.org/0000-0001-6580-7073
Universidade Federal do Piauí, Brasil
E-mail: palomasaraiva@ ufpi.edu.br
Júlio César Galdino de Sousa
ORCID: https://orcid.org/0000-0002-4126-6257
Universidade Federal do Piauí, Brasil
E-mail: jcgs@ ufpi.edu.br
Sammy Sidney Rocha Matias
Same

\section{Resumo}

As pastagens contribuem de maneira significativamente para os meios de subsistência e serviços ecossistêmicos e são consideradas como principal fonte de fibra e nutrientes animal. Este trabalho teve como objetivo avaliar a variabilidade espacial dos teores de cálcio, alumínio, acidez potencial, fósforo e carbono orgânico total, bem como sua correlação com a produtividade de uma pastagem de capim Mombaça em um Neossolo Litólico Distrófico no município de Floriano-PI. O trabalho foi realizado na fazenda experimental do Colégio Técnico de Floriano (CTF), pertencente à Universidade Federal do Piauí. A área avaliada corresponde a 2 ha cultivada com capim Mombaça (Panicum Maximum cv. Mombaça) irrigado com sistema de aspersão. A amostragem do solo foi realizada nos pontos de cruzamento de uma malha, com intervalos regulares de 10 metros em uma área de 0,54 ha, perfazendo o total de 54 pontos amostrais. Para análise dos dados foram utilizados os métodos da estatística clássica, análise de correlação, análise geoestatística e técnica de interpolação de dados. O diagnóstico da fertilidade do solo mostra o fósforo como o mais deficiente seguido do cálcio. Os valores de alumínio e acidez potencial mostram-se adequados ao bom desenvolvimento do capim Mombaça. A variabilidade dos mapas mostra a heterogeneidade da distribuição e concentração dos elementos no solo, o que possibilita a aplicação de fertilizantes em taxas variadas visando sua homogeneização. A produtividade teve forte relação com os teores cálcio e fósforo. $\mathrm{O}$ uso da geoestatísticas podem ser usadas para otimizar áreas de produção capim Mombaça em Neossolos Litólicos.

Palavras-chave: Geoestatística; Atributos químicos; Forrageiras.

\begin{abstract}
Rangelands contribute significantly to livelihoods and ecosystem services and are considered a major source of fiber and animal nutrients. This work was carried out in Floriano-PI with the objective of evaluating the spatial variability of calcium, aluminum, potential acidity, phosphorus and total organic carbon contents, as well as their correlation with the productivity of a Mombaça grass pasture in a Litholic Dystrophic Neosol. For data analysis, the methods of
\end{abstract}


classical statistics, correlation analysis, geostatistical analysis and data interpolation technique were used. The diagnosis of soil fertility shows phosphorus as the most deficient, followed by calcium. The aluminum values and potential acidity are adequate for the good development of Mombaça grass. The variability of the maps shows the heterogeneity of the distribution and concentration of elements in the soil, which makes it possible to apply fertilizers at different rates for its homogenization. Productivity had a strong relationship with calcium and phosphorus contents. The use of geostatistics can be used to optimize Mombaça grass production areas in Litholic Neosols.

Keywords: Geostatistics; Chemical attributes; Forage.

\section{Resumen}

Los pastizales contribuyen significativamente a los medios de vida y los servicios de los ecosistemas y se consideran una fuente importante de fibra y nutrientes animales. Este trabajo tuvo como objetivo evaluar la variabilidad espacial de calcio, aluminio, acidez potencial, fósforo y carbono orgánico total, así como su correlación con la productividad de un pasto de pasto Mombaça en un Neosol Litólico Distrófico en Floriano-PI. El trabajo se llevó a cabo en la finca experimental del Colégio Técnico de Floriano (CTF), perteneciente a la Universidad Federal de Piauí. El área evaluada corresponde a 2 ha cultivadas con pasto Mombaça (Panicum Maximum cv. Mombaça) regadas con sistema de aspersión. El muestreo de suelo se realizó en los puntos de cruce de una cuadrícula, a intervalos regulares de 10 metros en un área de 0.54 ha, para un total de 54 puntos de muestreo. Para el análisis de datos se utilizaron los métodos de estadística clásica, análisis de correlación, análisis geoestadístico y técnica de interpolación de datos. El diagnóstico de fertilidad del suelo muestra el fósforo como el más deficiente, seguido del calcio. Los valores de aluminio y la acidez potencial son adecuados para el buen desarrollo de la hierba Mombaça. La variabilidad de los mapas muestra la heterogeneidad de la distribución y concentración de elementos en el suelo, lo que permite aplicar fertilizantes a diferentes tasas para su homogeneización. La productividad tuvo una fuerte relación con los contenidos de calcio y fósforo. El uso de geoestadísticas se puede utilizar para optimizar las áreas de producción de pasto Mombaça en Litholic Neosoles.

Palabras clave: Geoestadística; Atributos químicos; Forrajes.

\section{Introdução}

A abertura de área para a implantação de pastagem para fornecimento de alimentos para o gado, representa em torno de 20-22\% da terra livre de gelo no globo terrestre (Phelps \& Kaplan, 2017). O uso da pastagem é extenso e amplamente distribuído de forma global, sendo estimado em cerca de 3 bilhões de hectares pela Organização das Nações Unidas para a Alimentação (FAO, 2019).

No Brasil as áreas com pastagens ocupam aproximada de $175 \mathrm{Mha}$, sendo uma das principais forma de uso da terra no pais (Brito et al., 2018). As pastagens quando manejadas de forma adequadas contribui de forma positiva para biota do solo. Rosa et al., (2015) Verificaram que os ambientes mais estáveis como floresta nativa, áreas reflorestadas com eucaliptos e pastagens perenes tende a favorecerem a biodiversidade do solo.

As pastagens ainda contribuem significativamente para os meios de subsistência e serviços nos ecossistêmicos (Fries and Rosenzweig, 2010), para a intensificação sustentável (Bogaerts et al., 2017; Palermo et al., 2014), potencial para preservação da terra, diversificação do uso da terra (Latawiec et al., 2014), mitigação da emissão de gases de efeito estufa (Cardoso et al., 2016; Silva et al., 2018) e expansão da bioenergia (Cai et al., 2011).

Os avanços tecnológicos na agropecuária demonstram a necessidade de mensurar a variação espacial da pastagem e dos atributos do solo, com o objetivo de melhorar o aproveitamento de recursos naturais e financeiros, uma vez que essa variabilidade espacial dos atributos químicos, físicos e biológicos do solo, influência a eficiência do manejo da pastagem e seu desenvolvimento (Montanari et al., 2013).

A variabilidade das produtividades das culturas acompanha a fertilidade do solo e, é imprescindível conhecer quais atributos exercem as maiores influências (Marques Júnior et al., 2000). Dessa forma, pesquisas têm sido desenvolvidas no sentido de estudar a correlação entre atributos do solo e produtividade das culturas (Montanari et al., 2012; Souza et al., 2010). Grego et al., (2012), estudando produtividade de pastagem e atributos químicos em Latossolo Vermelho Amarelo, encontraram correlação espacial entre a produção de biomassa e os atributos químicos do solo. 
A geoestatistica é uma importante ferramenta para entender as interações em um ecossistema de pastagem, reduzir possíveis causas de degradação e demonstrar a melhor alternativa de manejo para o complexo solo-planta-animal (Pariz et al., 2011).

Devido à intensa conversão de áreas de biomas nativos em áreas agrícolas é preocupante o cenário de degradação das áreas de produção. Neste sentido, é imprescindível a avaliação dos atributos do solo e da pastagem, visando manter e/ou melhorar a qualidade dos recursos naturais, principalmente do solo e da água, por meio de adoção de sistemas que visem uso eficiente e sustentável do solo e o incremento das qualidades químicas, físicas e biológicas.

Portanto, o objetivo do trabalho foi conhecer a variabilidade espacial dos teores de cálcio, alumínio, acidez potencial, fósforo e carbono orgânico total, bem como sua correlação com a produtividade de uma pastagem de capim Mombaça em um Neossolo Litólico Distrófico no município de Floriano-PI.

\section{Material e Métodos}

\section{Área de estudo}

O presente trabalho foi realizado na fazenda experimental do Colégio Técnico de Floriano (CTF), pertencente à

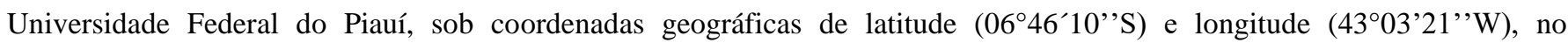
município de Floriano-PI (Figura 1). Segundo a classificação de Köppen, (1936), o clima é tropical semiárido, quente e úmido. Com base nos dados do período de 1995 a 2019, a temperatura média da área é de $28,1^{\circ} \mathrm{C}$, com valores médios da mínima e da máxima, respectivamente, de 23,1 e $34,2{ }^{\circ} \mathrm{C}$, com a umidade relativa média do ar de $64,2 \%$ e a precipitação pluviométrica média de 973,16 mm (INMET, 2020).

Figura 1 - Localização da área experimental e representação da malha de amostragem do solo.

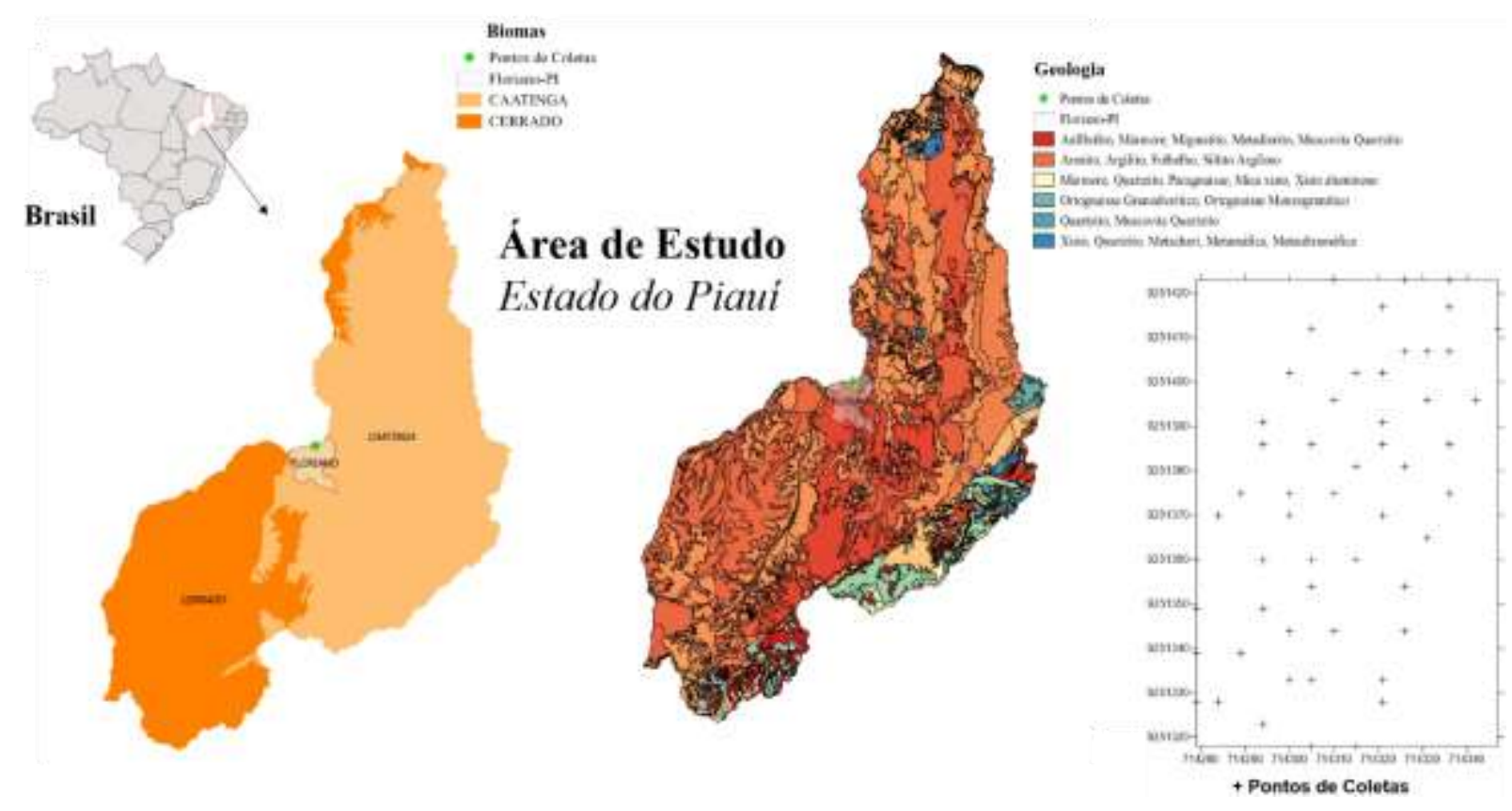

Fonte: Autores.

O clima da área apresenta duas estações bem definidas: uma seca (maio a setembro) e outra chuvosa (outubro a abril) (Gráfico 1). Os dados de precipitação e temperaturas média referentes ao período estudado encontram-se no Gráfico 1 (INMET, 2020). 
Gráfico 1 - Precipitação pluviométrica e temperatura do município de Floriano, PI no ano de 2019 (INMET, 2020).

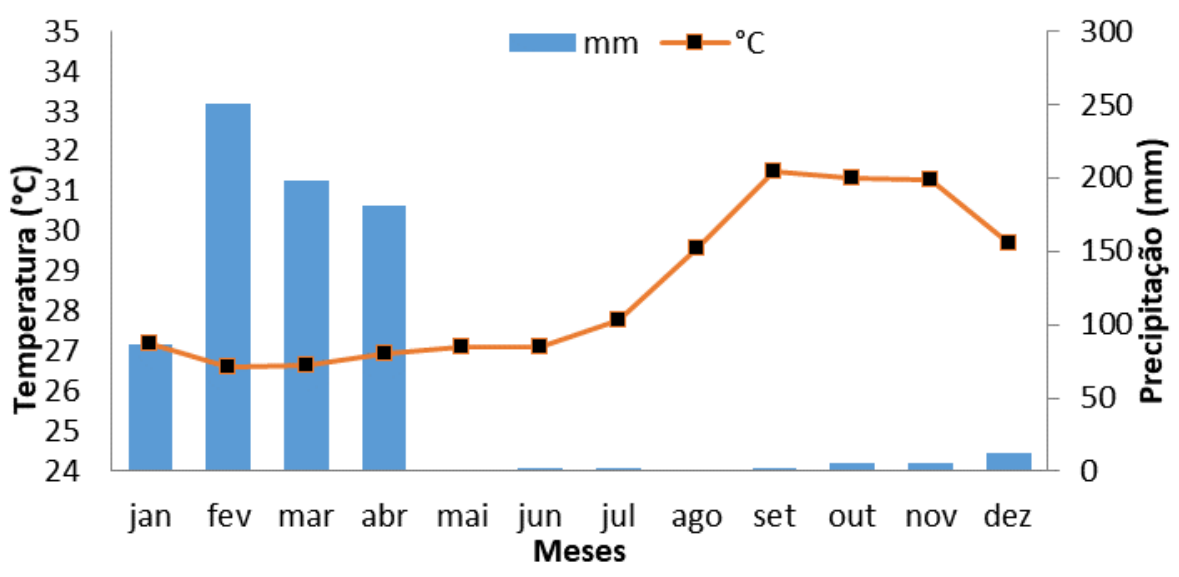

Fonte: Autores.

O solo da área experimental é classificado como Neossolo Litólico Distrófico de textura areia franca (Santos et al., 2018). As analises física e químicas do solo estão descrito na Tabela 1. As análises seguiram a metodologia de Teixeira et al., (2017).

Tabela 1 - Caracterização química na camada de 0-0,2 m do Neossolo Litólico Distrófico na área de capim-Mombaça.

\begin{tabular}{|c|c|c|c|c|c|c|c|c|}
\hline \multirow[t]{2}{*}{$\mathrm{pH} \mathrm{H_{2 } \mathrm { O }}$} & $\mathbf{K}$ & $\mathbf{C a}$ & Mg & Al & $\mathrm{H}+\mathrm{Al}$ & COT & Areia & Argila $\quad$ Ds $^{1}$ \\
\hline & $-\mathrm{mg} \mathrm{dm}^{-3}-$ & $-\ldots$ & $--\mathrm{cn}$ & $l_{c} \mathrm{dm}$ & -- & $\%$ & - - - - $\mathrm{g} \mathrm{kg}^{-1}$ & $\mathrm{~kg} \mathrm{dm}^{-3}$ \\
\hline 5.8 & 32.6 & 1.50 & 0.81 & 0.10 & 3.48 & 1.45 & 775 & 1,53 \\
\hline
\end{tabular}

${ }^{1}$ Ds: densidade do solo. Fonte: Autores.

A área avaliada corresponde a 2 ha cultivada com capim Mombaça (Panicum Maximum cv. Mombaça) irrigado com sistema de aspersão. A adubação foi feita com base na necessidade da cultura e atendendo as recomendações da análise de solo.

\section{Amostragem e variáveis avaliadas}

A amostragem do solo foi realizada nos pontos de cruzamento de uma malha, com intervalos regulares de 10 metros em uma área de 0,54 ha, perfazendo o total de 54 pontos amostrais. Em cada ponto da malha foi levantada a sua cota, com o auxílio de um teodolito e georreferenciados com GPS. Após a identificação dos pontos de coleta na área foram abertos miniperfis com 0,40 m de profundidade, 0,40 m de largura e 0,60 m de comprimento, nos quais coletou-se amostras deformadas nas profundidades de $0-0,2 \mathrm{~m}$.

Os teores de $\mathrm{Al}, \mathrm{Ca}$ e $\mathrm{Mg}$ trocáveis foram extraídos com $\mathrm{KCl} 1 \mathrm{~mol} \mathrm{~L}{ }^{-1}$ e a acidez potencial (H+Al) foi extraída com acetado de cálcio a pH 7, sendo estes parâmetros determinados posteriormente por titulometria (Teixeira et al., 2017). Os teores do P disponível foram extraídos por solução de Mehlich $^{-1}$ e determinado por colorimetria (Teixeira et al., 2017). O carbono orgânico total do solo (COT) foi determinado pelo método de Walkley; Black, (1934), no qual o COT foi oxidado usando $\mathrm{K}_{2} \mathrm{Cr}_{2} \mathrm{O}_{7}$ em um ambiente úmido e depois medido por titulação com sulfato de ferro e amônio 0,1 M.

Para quantificação em toneladas de massa seca produzida por hectare, foram coletados material vegetal de capim Mombaça presente uma área correspondente a $1,0 \mathrm{~m}^{2}$ em torno do ponto georreferenciado da malha amostral (Salman et al., 2006). Após a identificação dos pontos, foi feito o corte da pastagem com rebrote de 120 dias de forma manual, sendo o 
material coletado em cada ponto processado em laboratório e levado a estufa de circulação de ar forçada a $70{ }^{\circ} \mathrm{C}$ por 72 horas para retirar a umidade e posterior pesagem em balança de precisão.

\section{Análise dos dados}

Os atributos químicos do solo foram avaliados por meio da análise estatística descritiva, sendo calculados a média, mediana, variância, valores máximos e mínimos, e os coeficientes de variação, assimetria e curtose. A hipótese de normalidade dos dados será testada pelo teste de Kolmogorov-Smirnov, por meio do programa computacional SAS (Schlotzhaver \& Littell, 1997).

A dependência espacial foi analisada por meio de ajustes de semivariogramas (Vieira, 2000), com base na pressuposição de estacionariedade da hipótese intrínseca. Os ajustes dos modelos teóricos para o semivariograma foram estimados com base no efeito pepita $\left(\mathrm{C}_{0}\right)$, patamar $\left(\mathrm{C}_{0}+\mathrm{C}_{1}\right)$ e alcance (a). Para analisar o grau da dependência espacial dos atributos em estudo, será utilizado a classificação de Cambardella et al., (1994).

Os modelos de semivariogramas considerados foram o esférico, exponencial, linear e gaussiano, sendo ajustados por meio do programa GS+ (versão 7.0) (Gamma Design Software, 2018). Os modelos foram utilizados no desenvolvimento de mapas de isolinhas (krigagem). Em caso de dúvida entre mais de um modelo para o mesmo semivariograma, foi considerado o maior valor do coeficiente de correlação obtido pelo método de validação cruzada. Para elaboração dos mapas de distribuição espacial das variáveis, foi utilizado o programa Surfer (Goldem Software, 2020).

Nos mapas da distribuição espacial dos atributos químicos do solo os teores foram interpretados segundo descrição feita por Ribeiro; Guimarães; Alvarez, (1999), descrito na Tabela 2.

Tabela 2 - Interpretação dos resultados de análise de pH em água, potássio, fósforo, cálcio, magnésio, hidrogênio + alumínio, soma de bases, capacidade de troca catiônica e saturação por bases.

\begin{tabular}{|c|c|c|c|c|c|}
\hline Variáveis & \multicolumn{5}{|c|}{ Classificação Agronômica } \\
\hline \multirow{3}{*}{$\mathrm{pH}$ em $\mathrm{H}_{2} \mathrm{O}$} & Acidez muito elevada & Acidez elevada & Médio & Fraco & Alcalino \\
\hline & $<4.5$ & $4.5-50$ & $5.1-6.0$ & $6.1-6.9$ & $>7.0$ \\
\hline & $\begin{array}{l}\text { Muito } \\
\text { baixo }\end{array}$ & Baixo & Médio & Bom & Muito bom \\
\hline $\mathrm{K}^{+} \mathrm{cmol}_{\mathrm{c}} \mathrm{dm}^{-3}$ & $<0.50$ & $0.5-1.0$ & $1.0-2.0$ & ---- & $>2.00$ \\
\hline $\mathrm{P} \mathrm{mg} \mathrm{dm}^{-3}$ & $<8.0$ & $8.1-11.4$ & $11.5-15.8$ & $15.9-24.00$ & $>24.00$ \\
\hline $\mathrm{Ca}^{2+} \mathrm{cmol}_{\mathrm{c}} \mathrm{dm}^{-3}$ & 0.40 & $0.41-1.20$ & $1.21-2.40$ & $2.41-4.00$ & $>4.00$ \\
\hline $\mathrm{Mg}^{2+} \mathrm{cmol}_{\mathrm{c}} \mathrm{dm}^{-3}$ & 0.15 & $0.16-0.45$ & $0.46-0.90$ & $0.91-1.50$ & $>1.50$ \\
\hline $\mathrm{H}+\mathrm{Al} \mathrm{cmol}_{\mathrm{c}} \mathrm{dm}^{-3}$ & 1.00 & $1.01-2.50$ & $2.51-5.00$ & $5.01-9.00$ & $>9.00$ \\
\hline $\mathrm{SB} \mathrm{cmol}_{\mathrm{c}} \mathrm{dm}^{-3}$ & 0.60 & $0.61-1.80$ & $1.81-3.60$ & $3.61-6.00$ & $>6.00$ \\
\hline CTC cmolc $_{\mathrm{c}} \mathrm{dm}^{-3}$ & 1.60 & $1.61-4.30$ & $4.31-8.60$ & $8.61-15.00$ & $>15.00$ \\
\hline $\mathrm{V} \%$ & 20.0 & $20.1-40.0$ & $40.1-60.0$ & - & - \\
\hline
\end{tabular}

Fonte: Ribeiro, Guimarães e Alvarez (1999).

Para avaliar a correlação entre os atributos químicos e a produtividade foi realizado a correlação de Pearson, utilizando o pacote estatístico R (R Core Team, 2020).

\section{Resultados e Discussão}

\section{Estatística descritiva}

Na Tabela 3 são apresentados os resultados da análise descritiva das propriedades químicas do solo. Os valores de média e mediana apresentaram próximos, mostrando que a média não foi influenciada por valores discrepantes (Rodrigues et 
al., 2017). Entretanto, apenas alumínio e produtividade apresentaram distribuição normal pelo teste de Kolmogorov-Sminov a $5 \%$ (Tabela 3$)$.

Tabela 3 - Estatística descritiva de atributos químicos, carbono orgânico e produtividade de pastagem de capim Mombaça.

\begin{tabular}{|c|c|c|c|c|c|c|c|}
\hline Estatística descritiva & $\begin{array}{c}\mathrm{Ca}^{2+} \\
----\end{array}$ & $\begin{array}{c}\mathbf{M g}^{2+} \\
---(\mathrm{cm} \\
\end{array}$ & $\begin{array}{c}\mathbf{A l}^{3+} \\
\left.\mathrm{dm}^{-3}\right)--\end{array}$ & 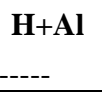 & $\begin{array}{c}\mathbf{P} \\
\left(\mathrm{mg} \cdot \mathrm{dm}^{-3}\right)\end{array}$ & $\begin{array}{c}\text { COT } \\
\left(\mathrm{g} \cdot \mathrm{kg}^{-1}\right) \\
\end{array}$ & $\begin{array}{c}\mathbf{P F}^{\mathbf{1}} \\
\left(\mathrm{Mg} \mathrm{ha}^{-1}\right)\end{array}$ \\
\hline Média & 1.10 & 1.07 & 0.05 & 1.51 & 1.27 & 1.09 & 0.7 \\
\hline Mediana & 1.2 & 1.05 & 0.00 & 1.48 & 1.24 & 1.05 & 0.55 \\
\hline Erro padrão & 0.05 & 0.05 & 0.01 & 0.07 & 0.08 & 0.08 & 0.07 \\
\hline Variância simples & 0.15 & 0.15 & 0.004 & 0.29 & 0.39 & 0.35 & 0.29 \\
\hline Mínimo & 0.40 & 0.40 & 0.00 & 0.33 & 0.36 & 0.23 & 0.20 \\
\hline Máximo & 1.90 & 1.90 & 0.20 & 2.64 & 3.24 & 2.63 & 2.95 \\
\hline Coeficiente de variação (\%) & 34.68 & 36.29 & 85.37 & 35.71 & 49.17 & 54.58 & 77.33 \\
\hline Coeficiente de assimetria & -0.10 & 0.35 & 0.99 & 0.02 & 0.77 & 0.66 & 2.95 \\
\hline Coeficiente de curtose & -0.66 & -0.80 & -0.12 & -0.19 & 0.56 & -0.27 & 9.78 \\
\hline Kolmogorov-Sminov (p valor) & $0.12^{\text {ns }}$ & $0.14^{\mathrm{ns}}$ & $0.39 *$ & $0.10^{\mathrm{ns}}$ & $0.09^{\mathrm{ns}}$ & $0.11^{\mathrm{ns}}$ & $0.18^{*}$ \\
\hline
\end{tabular}

*Distribuição normal pelo teste de Komogorov-Sminov p $\leq$ 0,05.; ${ }^{1} \mathrm{PF}$ : Produtividade de fitomassa do capim Mombaça. Fonte: Autores.

Os atributos químicos $\mathrm{Ca}, \mathrm{Mg}$ e $\mathrm{H}+\mathrm{Al}$ apresentaram os menores coeficientes de variação, embora acima de $34 \%$, revelando heterogeneidade; o $\mathrm{Al}$ apresentou o maior coeficiente de variação seguido pela produtividade (CV> 60\%). Corroborando com Barbieri et al. (2017), ao conduzirem experimento em área com pastagem degradada, encontram alta variabilidade para os atributos químicos do solo, com destaque para o $\mathrm{Al}(235,70 \%)$.

O coeficiente de variação elevado, é explicado em trabalhos como os de Matias et al., (2015), e Sanches et al., (2020), que atribuem a alta variabilidade dos atributos químicos do solo as interações dos processos de formação do solo e das práticas de manejo, como, a aplicação de corretivos e fertilizantes e a zonas de baixa fertilidade, influenciados principalmente pela dinâmica da água em áreas convexas que reduz a capacidade de manutenção de nutrientes no solo.

A aplicação da geoestatística é justificada pela variabilidade dos dados, onde utiliza-se desta forma, os coeficientes de assimetria e curtose para a avaliação da normalidade dos dados. O coeficiente de assimetria é utilizado na caracterização de como e quando as frequências se afastam da simetria sendo indicado que valores próximos do valor central zero (Cortez et al., 2011).

A assimetria é uma medida de distribuição de probabilidade de uma variável aleatória, onde o valor zero indica distribuição simétrica em ambos os lados da média. Quando os valores são positivos a distribuição é assimétrica à direita e se negativo a distribuição é assimétrica à esquerda (Tripathi et al., 2015). Os valores de assimetria dos atributos avaliados apresentaram distribuição assimétrica positiva (médias maiores que a mediana), com exceção para Ca, embora todos os próximos de zero, ocasionadas pelos valores de média e mediana próximos entre si no qual tendem a simetria. Silva et al., (2020), ao estudarem a variabilidade espacial da fertilidade em área cultivada com capim Tifton 85, encontram valores positivos para $\mathrm{P}, \mathrm{Ca}, \mathrm{Mg}, \mathrm{Al}$ e $\mathrm{H}+\mathrm{Al}$, e valor negativo para COT.

A curtose serve como indicativo das distribuições dos valores analisados no qual verifica o grau de achatamento da distribuição da frequência de um conjunto de dados (Lima et al., 2010). Os coeficientes de curtose para todos os atributos mantiveram-se próximos a zero, exceto produtividade (curtose $=9,78$ ), no qual ultrapassa os valores aceitos entre -2 e +2 (Negreiros Neto et al., 2014). 


\section{Geoestatística}

Os resultados da geoestatística demonstram que os atributos químicos e a produtividade de fitomassa apresentaram dependência espacial (Tabela 4). A maioria das variáveis ( $\mathrm{Mg}, \mathrm{Al}, \mathrm{H}+\mathrm{Al}, \mathrm{P}$ e PF) ajustaram-se ao modelo exponencial, comumente encontrados na representação dos atributos do solo nas camadas superficiais (Aquino et al., 2014; Dalchiavon et al., 2012; Negreiros Neto et al., 2014; Santana da Silva Carneiro et al., 2016). Macedo Neto et al., (2020), destacaram que os modelos exponencial e gaussiano se ajustam com maior frequência para as variáveis de fertilidade do solo e apresentam coeficiente de determinação do modelo $\left(\mathrm{R}^{2}\right)$ com valores altos, corroborando com os resultados encontrados nesse trabalho.

Tabela 4 - Modelos e parâmetros estimados por semivariogramas experimentais para atributos químicos, carbono orgânico e produtividade de pastagem.

\begin{tabular}{lccccccc}
\hline \multicolumn{1}{c}{ Parâmetros } & Ca & Mg & Al & H+Al & P & COT & PF \\
\hline Modelo & ESF & EXP & EXP & EXP & EXP & GAU & EXP \\
Efeito pepita (C) & 0.00010 & 0.0434 & 0.00034 & 0.0300 & 0.0350 & 0.0010 & 0.00032 \\
Patamar $\left(C_{0}+\right.$ C) & 0.22020 & 0.2808 & 0.00836 & 0.4070 & 0.9670 & 0.4690 & 0.00085 \\
GDE (\%) & 0.04 & 15.45 & 4.07 & 7.37 & 3.61 & 0.21 & 37.65 \\
$\mathrm{R}^{2}$ & 0.711 & 0.890 & 0.931 & 0.890 & 0.707 & 0.994 & 0.948 \\
SQR & $4.9 .10^{-5}$ & $1.4 .10^{-3}$ & $3.4 .10^{-8}$ & $9.0 .10^{-5}$ & 0.0197 & $7.7 .10^{-4}$ & $3.7 .10^{-9}$ \\
A (m) & 17.40 & 25.20 & 18.60 & 7.35 & 15.54 & 17.53 & 74.61 \\
\hline
\end{tabular}

ESF: Esférico; GAU: Gaussiano; EXP: Exponencial; GDE: Grau de Dependência Espacial $\left[\mathrm{C}_{0} /\left(\mathrm{C}_{0}+\mathrm{C}_{1}\right) \cdot 100\right]$; $\mathrm{R}^{2}$ : Coeficiente de determinação do modelo; SQR: Soma dos quadrados dos resíduos; A: Alcance; PF: Produtividade de fitomassa do capim Mombaça. Fonte: Autores.

O efeito pepita indica a variabilidade não explicada geralmente associadas a erros de medida e micro variações não detectadas, considerando a distância de amostragem utilizada (Mantovanelli et al., 2015). Os modelos ajustados não apresentaram valores altos para efeito pepita.

O GDE, expresso pela razão entre o efeito pepita $\left(C_{0}\right)$ e o patamar $\left(C_{0}+C_{1}\right)$, foi utilizado segundo classificação de Cambardella et al. 1994. A análise dos dados mostrou forte dependência para os atributos químicos do solo (GDE < 25\%) e moderado para produtividade $(25 \%<\mathrm{GDE} \leq 75 \%)$. O forte GDE, indica que o modelo foi capaz de detectar a variabilidade existente no local, sugerindo que os atributos do solo não estão dispostos no espaço de forma aleatória e que esses atributos são influenciados por fatores intrínsecos do solo, como material de origem, mineralogia, relevo, organismos e tempo (OLIVEIRA, et al., 2021; VOGADO et al., 2020) alterando-se menos com os valores extrínsecos, tais como, adubação e correção, que por sua vez não influenciam as respostas do variogramas (Aquino et al., 2014).

O alcance leva ao entendimento sobre a distância máxima em que um ponto amostral sofre interferência do outro ponto em uma variável qualquer, ou seja, quando maior o alcance, mais homogênea é a distribuição da variável na área de estudo (Vogado et al., 2020). A produtividade de fitomassa do capim Mombaça, apresentou maior homogeneidade espacial, pois exibiu o valor mais elevado de alcance. E o menor alcance foi encontrado para $\mathrm{H}+\mathrm{Al}$ (7,35 m), mostrando-se inferior a distância entre os pontos amostrados, corroborando com os resultados encontrados por Santana et al. (2019).

Mediante ajustes dos semivariogramas, foi possível a elaboração dos mapas com as devidas interpolações dos valores no campo estudado possibilitando estimar os valores não amostrados (Figura 2). 
Figura 2 - Mapas de isolinhas de cálcio (A), magnésio (B), alumínio (C), acidez potencial (D), fósforo (E), COT (F) e Produtividade $(\mathrm{G})$ em área de capim Mombaça.

(A)

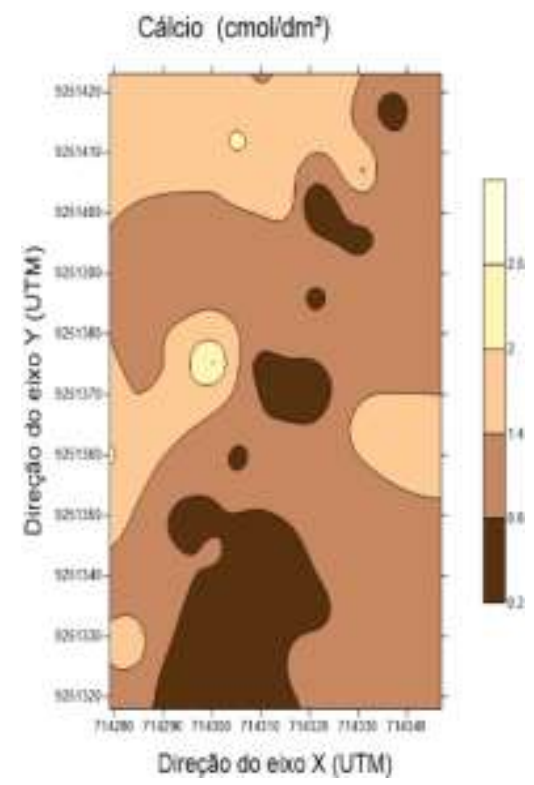

(C)

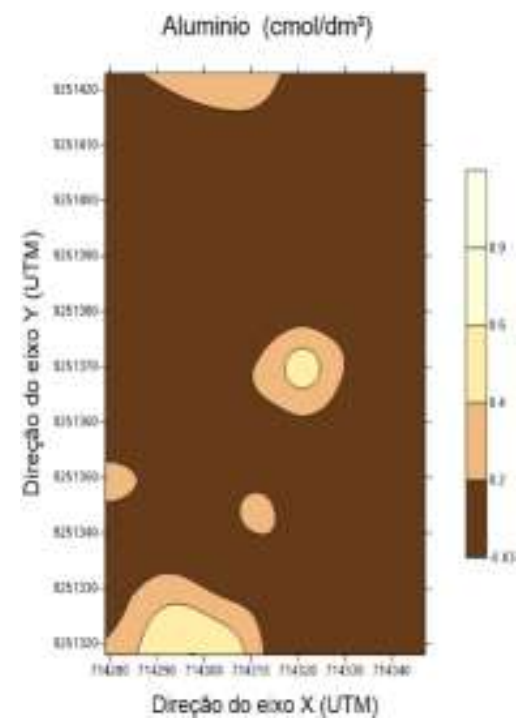

(E)
(B)

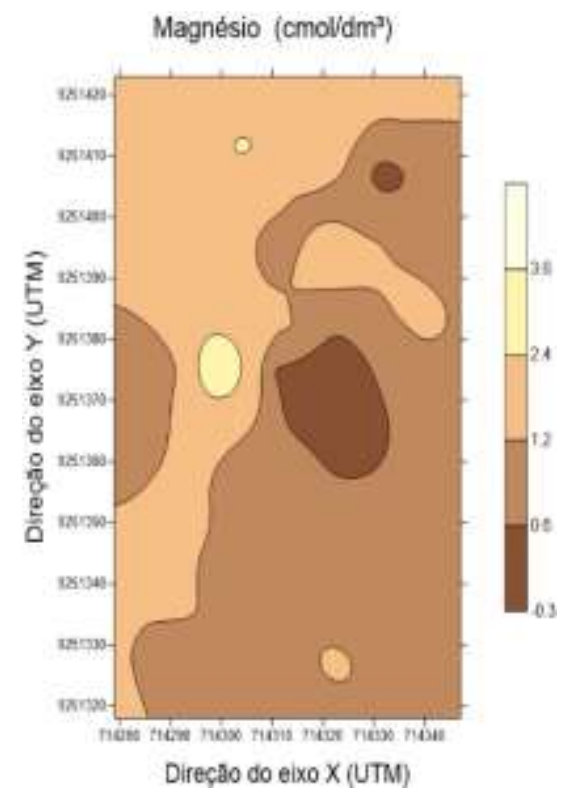

(D)

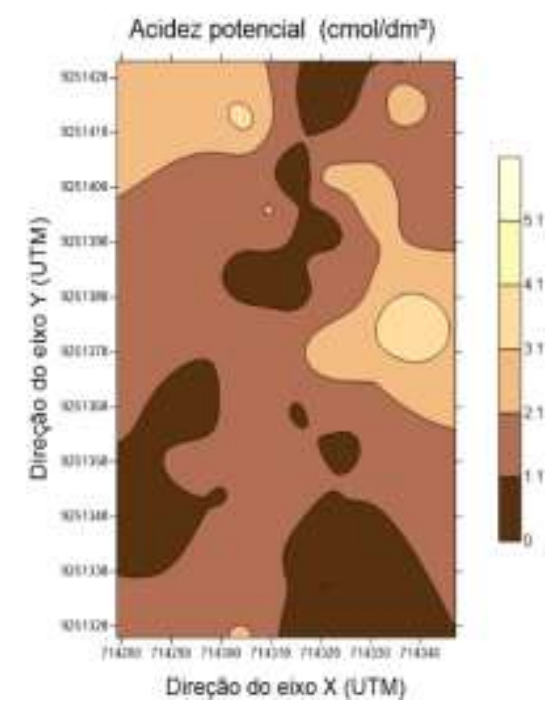

(F) 

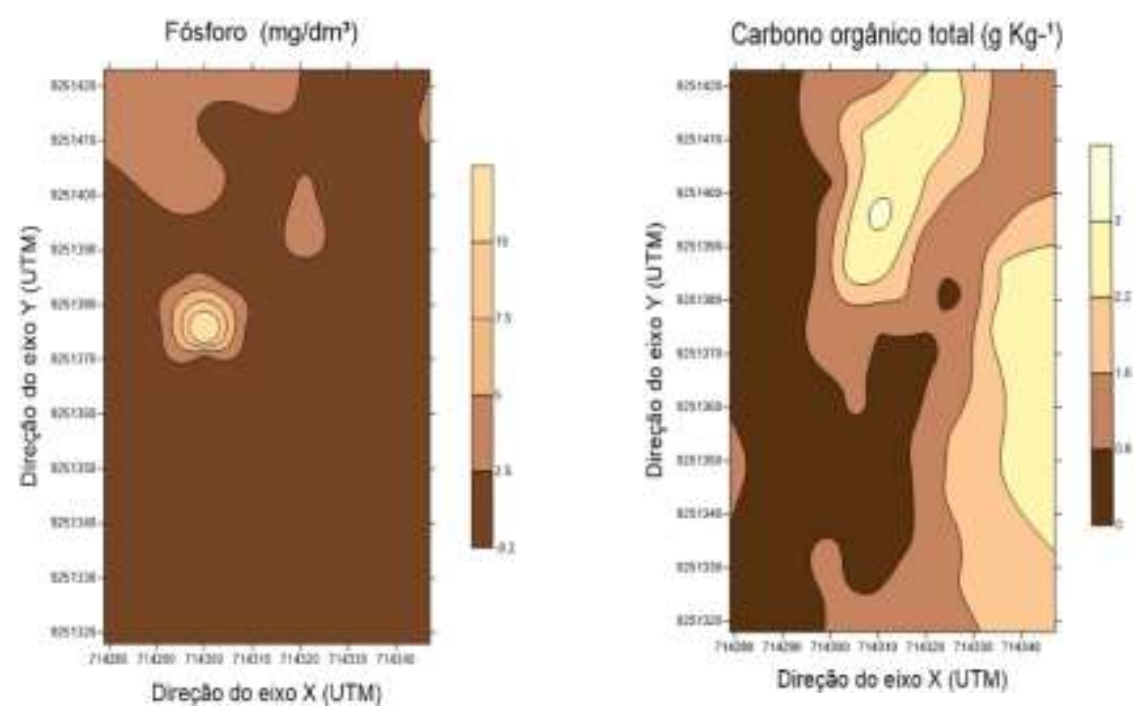

(G)

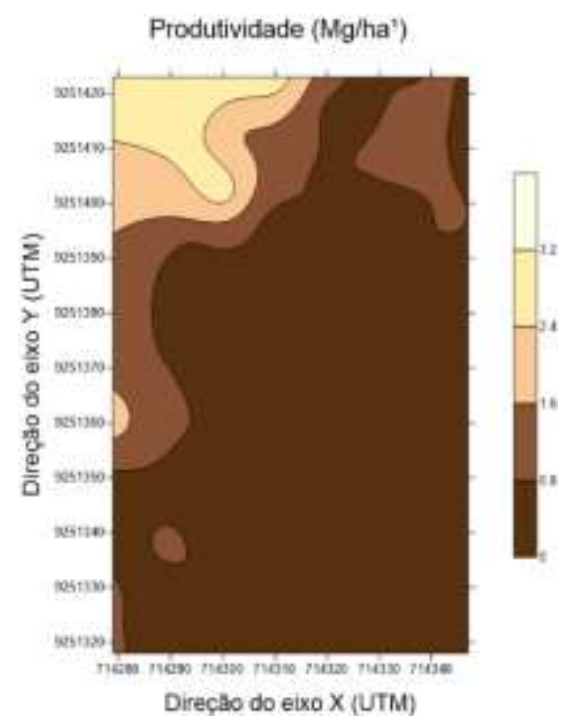

Fonte: Autores.

Com relação aos mapas apresentados, foi possível visualizar grande variação dos atributos avaliados na área, confirmando os coeficientes de variação (Barbieri et al., 2020). Os valores de Ca mais representativos encontraram-se na faixa de 0.8 a $1.4 \mathrm{cmol}_{\mathrm{c}} \mathrm{dm}^{-3}$ (Figura 2a) apresentando assim, valores considerados baixos (Tabela 2). Os valores mais elevados de $\mathrm{Ca}\left(1.4\right.$ a $\left.2.0 \mathrm{cmol}_{\mathrm{c}} \mathrm{dm}^{-3}\right)$ concentrados em áreas marginais do mapa, podem estar relacionados a aplicação inadequada de calcário. Carvalho et al. (2018), observaram as concentrações de Ca (Figura 2a) e Al (Figura 2c), e constataram que são antagônicas, pois, onde a concentração de $\mathrm{Al}$ é maior ocorrem os menores teores de $\mathrm{Ca}$, o que é perceptível neste trabalho.

Para teores de $\mathrm{Mg}$, os valores variaram entre 0.6 a $1.2 \mathrm{cmol} \mathrm{dm}^{-3}$ (Figura 2b), sendo classificados como médios a bons (Tabela 2); uma outra parte considerável da área expressou valores entre 1.2 a $2.4 \mathrm{cmol}_{\mathrm{c}} \mathrm{dm}^{-3}$ enquadrando-se em muito bom (Tabela 2), diferente do que foi observado para Ca, o solo mostrou-se com níveis condizentes de Mg para manutenção da pastagem. O Mg tem um papel importante na fisiologia das plantas, é responsável por aumentar a tolerância a diferentes tipos de estresses, em que a cultura indica as mudanças na demanda de Mg sob condições adversas de crescimento (Gransee \& Führs, 2012).

Em relação ao Al, Ribeiro et al. (1999), relatam que o valor máximo de saturação tolerado pelo capim Mombaça é de 25\%. O mapa apresentado neste trabalho (Figura 2c) mostra uma continuidade na espacialização do Al, em que, em níveis 
mantiveram-se baixos e adequados ao desenvolvimento da pastagem, variando entre 0 e $0.2 \mathrm{cmol}_{\mathrm{c}} \mathrm{dm}^{-3}$, dessa forma, indicando baixo potencial de problemas relativos à toxicidade por alumínio. $\mathrm{O} \mathrm{pH}$ (Tabela 1) em nível apropriado, faz com que formas trocáveis de alumínio se modifiquem para hidróxido de alumínio, forma essa mais insolúvel, o que reduz sua atividade.

A acidez potencial $(\mathrm{H}+\mathrm{Al})$ mostrou descontinuidade espacial evidenciada pelo baixo valor de alcance (Tabela 4); o mapa apresentou maior concentração na faixa de 1.1 a $2.1 \mathrm{cmol}_{\mathrm{c}} \mathrm{dm}^{-3}$ (Figura 2d), classificado como baixo (Tabela 3). Os baixos valores de $\mathrm{H}+\mathrm{Al}$ denotam potencial limitado na liberar $\mathrm{H}^{+}$na solução do solo. Os locais que apresentam maior capacidade de acidificação, tendem a se correlacionar com as áreas de maior concentração de carbono orgânica (Santana et al., 2019); é possível ser observado na tabela 5 a correlação positiva entre H+Al e COT. Valores tidos como médios (2.51 - 5.00 $\mathrm{cmol}_{\mathrm{c}} \mathrm{dm}^{-3}$ ) (Tabela 3), indicam que a área cultivada não apresenta toxicidade, assim, não prejudicando o desenvolvimento do sistema radicular (Oliveira et al., 2021).

Os níveis de $\mathrm{P}$, encontraram-se majoritariamente entre 0 e $2.5 \mathrm{mg} \mathrm{dm}^{3}$ (Figura 2e), considerados como muito baixos (Tabela 3). Apesar da adubação fosfatada, conforme a necessidade da pastagem, o mapa é claro quanto a baixa mobilidade do $\mathrm{P}$ e sua alta fixação aos coloides do solo. Assim, apontando necessidade a aplicação de $90 \mathrm{~kg} \mathrm{ha}^{-1}$ de $\mathrm{P}_{2} \mathrm{O}_{5}$ em decorrência a análise de solo e exigência da espécie forrageira.

Os teores de COT encontrados, são muito baixos para todos os valores distribuídos. Os maiores fragmentos do mapa foram predominantemente compreendidos entre 0 a $0.8 \mathrm{~g} / \mathrm{kg}^{-1}$ e de 0.8 a $1.6 \mathrm{~g} \mathrm{~kg}^{-1}$ (Figura 2f). Barbieri et al. (2020) também encontraram resultados semelhantes na avaliação da variabilidade espacial em Neossolos embora outros trabalhos mostram que os valores de carbono para a mesma classe de solos avaliadas estão altos (VANSHCHENKO et al., 2007; MARTINS et al., 2019). Desta forma, o sistema de preparo na área estudada pode ter contribuído para a redução do COT, pelo aumento na taxa de decomposição da matéria orgânico (Freitas et al., 2018). O uso dos Neossolos Litólicos por ocorrerem em relevos acidentados, necessitam de cuidados, de preferência com tratos mais conservacionistas (Neves et al., 2011).

O capim Mombaça (Panicum maximum Jacq.) é considerado uma das forrageiras tropicais mais produtivas à disposição dos pecuaristas, podendo atingir produção de massa seca anual em torno de $33 \mathrm{t} \mathrm{ha}^{-1}$ (Jank, 1995). Os valores médios encontrados para o capim Mombaça no trabalho de Mombach et al. (2019) foi de $2.756 \mathrm{~kg}$ de massa seca por hectare, próximo ao valor máximo encontrado neste estudo (Tabela 3).

A produção de massa seca foi abaixo dos encontrados na literatura (Bittar, 2017; Brito et al., 2020), pode ter sido influenciado pela data da colheita experimental (Ferreira et al., 2008) ou pela baixa fertilidade do solos já que a variedade do capim necessita de 50 a 60\% de saturação por bases e possuir alto grau de exigência nutricional (Martha Júnior et al., 2007)

A análise de correlação linear simples entre os atributos químicos dos solos estudados e a produtividade constam na Tabela 5. O Ca e Mg apresentaram correlação negativa para H+ Al. A mesma relação foram encontradas nos trabalhos de Zucoloto et al. (2011), Silva Júnior et al. (2012) e (Maraschin et al., 2020). A adição de bases ao solo promove aumento da concentração de hidroxila onde uma parte reage com o excesso de $\mathrm{H}^{+}$da solução, reduzindo os teores de acidez potencial, e a outra parte precipita o alumínio em composto não tóxico (Oliveira \& Pavan, 1996). 
Tabela 5 - Correlação linear de Pearson entre a produtividade de pastagem e atributos químicos em um Neossolo.

\begin{tabular}{cccccccc}
\hline Variáveis & $\mathrm{Ca}$ & $\mathrm{Mg}$ & $\mathrm{Al}$ & $\mathrm{H}+\mathrm{Al}$ & $\mathrm{P}$ & $\mathrm{COT}$ & Produtividade \\
\hline $\mathrm{Ca}$ & $\mathbf{1}$ & 0,183 & $-0,099$ & $\mathbf{- 0 , 3 1 6 ^ { * }}$ & $\mathbf{0 , 3 2 6 ^ { * }}$ & $\mathbf{0 , 3 5 1 ^ { * }}$ & $\mathbf{0 , 2 9 5 *}$ \\
$\mathrm{Mg}$ & & $\mathbf{1}$ & 0,060 & $\mathbf{- 0 , 3 2 1 ^ { * }}$ & 0,232 & 0,018 & 0,142 \\
$\mathrm{Al}$ & & & $\mathbf{1}$ & 0,096 & $-0,017$ & $-0,137$ & 0,167 \\
$\mathrm{H}+\mathrm{Al}$ & & & & $\mathbf{1}$ & $-0,214$ & 0,072 & $-0,013$ \\
$\mathrm{P}$ & & & & & $\mathbf{1}$ & 0,160 & $\mathbf{0 , 5 2 4}$ \\
$\mathrm{COT}$ & & & & & & $\mathbf{1}$ & $-0,034$ \\
Prod. & & & & & & $\mathbf{1}$ \\
\hline
\end{tabular}

*Significativo $\mathrm{p} \leq 0,05$. Fonte: Autores.

Na Tabela 5 são evidente a correlação positiva entre Ca e os atributos P, COT e produtividade. Portz et al. (2015), viram que a adição de calcário no crescimento do capim Mombaça foi capaz de disponibilizar P ao solo. Maraschin et al., (2020) também obtiveram resultados positiva nos teores de P disponíveis com aplicação de calcário. Alvarado e Cajuste (1993), afirmam que isso ocorre pela redução da força de ligação do fósforo aos coloides do solo de acordo a substituição por outros elementos na solução.

Ferreira et al. (2013) observaram grande afinidade entre o Ca e o conteúdo de C do solo, demonstrando que esse comportamento pode promover melhorias da fertilidade do solo e causar impacto ambiental positivo pela atuação de dreno de C no sistema. A resposta de uma pastagem de Panicum maximum cv. Tobiatã a doses de calcário resultaram em aumento de produtividade (Martha Júnior et al., 2007).

Houve uma interação positiva entre $\mathrm{P}$ e produtividade, bem evidente ao observar os mapas da krigagem (Figura 2). A adubação fosfatara conforme a demanda da pastagem é necessária para manter o rendimento de matéria seca (Somavilla et al., 2021). E Magalhães et al., (2020), além de constatarem maior produção de matéria seca da parte aérea, também observaram incremento nos teores foliares de P; e que após a adubação fosfatada perceberam alteração nos valores de potássio, cálcio é magnésio do solo.

\section{Conclusão}

O diagnóstico da fertilidade do solo mostra o fósforo como o mais deficiente seguido do cálcio. Os valores de alumínio e acidez potencial mostram-se adequados ao bom desenvolvimento do capim Mombaça.

A variabilidade dos mapas mostra a heterogeneidade da distribuição e concentração dos elementos no solo, o que possibilita a aplicação de fertilizantes em taxas variadas visando sua homogeneização.

A produtividade teve forte relação com os teores cálcio e fósforo. O uso da geoestatísticas pode otimizar áreas de produção capim Mombaça em Neossolos Litólicos.

\section{Agradecimentos}

A Universidade Federal do Piauí (UFPI) e ao Colégio Técnico de Floriano (CTF) pelo apoio financeiro que permitiu realizar a pesquisa.

\section{Referências}

Alvarado, J., \& Cajuste, L. J. (1993). Encalado y Retencíon de Fósforo en Suelos Derivados de Cenizas Volcánicas. Turrialba, 43(4), $235-241$. 
Aquino, R. E., Marques Júnior, J., Campos, M. C. C., Oliveira, I. A., \& Siqueira, D. S. (2014). Distribuição espacial de atributos químicos do solo em área de pastagem e floresta. Pesquisa Agropecuária Tropical, 44(1), 32-41. https://doi.org/10.1590/s1983-40632014000100001

Barbieri, R. S., Unda, S. A. B., Carrillo, J. E. C., González, A. P., \& Montanari, R. (2020). Distribuição espacial de atributos do solo na região de El Oro, Equador. Colloquium Agrariae, 16(4), 46-60. https://doi.org/10.5747/ca.2020.v16.n4.a382

Bittar, D. Y. (2017). Características morfológicas e acúmulo de biomassa de forrageiras irrigadas em ambiente de domínio de cerrado. Instituto Federal De Educação, Ciência e Tecnologia Goiano. 100p.

Bogaerts, M., Cirhigiri, L., Robinson, I., Rodkin, M., Hajjar, R., Costa Junior, C., \& Newton, P. (2017). Climate change mitigation through intensified pasture management: Estimating greenhouse gas emissions on cattle farms in the Brazilian Amazon. Journal of Cleaner Production, 162, 1539-1550. https://doi.org/10.1016/j.jclepro.2017.06.130

Brito, J. L. S., Arantes, A. E., Ferreira, L. G., \& Sano, E. E. (2018). MODIS estimates of pasture productivity in the Cerrado based on ground and Landsat-8 data extrapolations. Journal of Applied Remote Sensing, 12(02), 1. https://doi.org/10.1117/1.jrs.12.026006

Cai, X., Zhang, X., \& Wang, D. (2011). Land availability for biofuel production. Environmental Science and Technology, 45(1), 334-339. https://doi.org/10.1021/es103338e

Cambardella, C. A., Moorman, T. B., Novak, J. M., Parkin, T. B., Karlen, D. L., Turco, R. F., \& Konopka, A. E. (1994). Field-Scale Variability of Soil Properties in Central Iowa Soils. Soil Science Society of America Journal, 58(5), 1501-1511. https://doi.org/10.2136/sssaj1994.03615995005800050033x

Cardoso, A. S., Berndt, A., Leytem, A., Alves, B. J. R., Carvalho, I. N. O., Soares, L. H. B., Urquiaga, S., \& Boddey, R. M. (2016). Impact of the intensification of beef production in Brazil on greenhouse gas emissions and land use. Agricultural Systems, 143, 86-96. https://doi.org/10.1016/j.agsy.2015.12.007

Cortez, J. W., Dantas, A., Moura, R. D., Olszevski, N., \& Jesus, H. (2011). Atributos físicos do argissolo amarelo do semiárido nordestino sob sistemas de preparo ( 1 ). 1, 1207-1216.

Rosa, M. G., Klauberg Filho, O., Bartz, M. L. C., Mafra, Á. L., De Sousa, J. P. F. A., \& Baretta, D. (2015). Macrofauna edáfica e atributos físicos e químicos em sistemas de uso do solo no planalto Catarinense. Revista Brasileira de Ciencia Do Solo, 39(6), 1544-1553. https://doi.org/10.1590/01000683rbcs20150033

Dalchiavon, F. C., Carvalho, M. P., Andreotti, M., \& Montanari, R. (2012). Variabilidade espacial de atributos da fertilidade de um Latossolo Vermelho Distroférrico sob Sistema Plantio Direto. Revista Ciência Agronômica, 43(3), 453-461. https://doi.org/10.1590/s1806-66902012000300006

Freitas, L., Oliveira, I. A., Casagrande, J. C., Silva, L. S., \& Campos, M. C. C. (2018). Latosols (Oxisols) carbon storage in natural and altered managements. Ciencia Florestal, 28(1), 228-239. https://doi.org/10.5902/1980509831575

Oliveira, E. L., \& Pavan, M. A. (1996). Control of soil acidity in no-tillage system for soybean production. Soil and Tillage Research, 38(1-2), 47-57. https://doi.org/10.1016/0167-1987(96)01021-5

Silva, R. O., Barioni, L. G., Pellegrino, G. Q., \& Moran, D. (2018). The role of agricultural intensification in Brazil's Nationally Determined Contribution on emissions mitigation. Agricultural Systems, 161, 102-112. https://doi.org/10.1016/j.agsy.2018.01.003

Fries, R., \& Rosenzweig, C. (2010). Toward a whole-landscape approach for sustainable land use in the tropics. Proceedings of the National Academy of Sciences of the United States of America, 107(46), 19627-19632. https://doi.org/10.1073/pnas.1011163107

FAO. (2019). Food and Agriculture Organization of the United Nations. https://coin.fao.org/coin-static/cms/media/5/13777480 671310/20130823_tor_national_me_adviser-osro103.pdf

Ferreira, A. O., Amado, T. J. C., Nora, D. D., Keller, C., \& Bortolotto, R. P. (2013). Mudança no conteúdo de cardono en calcio em latossolo melhorado por gesso e calcário no Rio Grande Do Sul. In Ciencia del Suelo (Vol. 31, Issue 1).

Ferreira, E. M., Santos, A. C., De Araújo, L. C., \& Cunha, O. F. R. (2008). Características agronômicas do Panicum maximum cv. "Mombaça" submetido a níveis crescentes de fósforo. Ciencia Rural, 38(2), 484-491. https://doi.org/10.1590/S0103-84782008000200030

GOLDEM SOFTWARE, I. (2020). Surfer ${ }^{\circledR} \mid 2 D \& 3 D$ mapping, modeling \& analysis software for scientists and engineers. https://www.goldensoftware.com/products/surfer

Gransee, A., \& Führs, H. (2012). Magnesium mobility in soils as a challenge for soil and plant analysis, magnesium fertilization and root uptake under adverse growth conditions. Plant and Soil 2012 368:1,368(1), 5-21. https://doi.org/10.1007/S11104-012-1567-Y

Grego, C. R., Rodrigues, C. A. G., Nogueira, S. F., Gimenes, F. M. A., Oliveira, A., Almeida, C. G. F., Furtado, A. L. S., \& Demarchi, J. J. A. A. (2012). Variabilidade espacial do solo e da biomassa epígea de pastagem, identificada por meio de geostatística. Pesquisa Agropecuária Brasileira, 47(9), 1404-1412. https://doi.org/10.1590/S0100-204X2012000900026

INMET. (2020). Instituto Nacional de Meteorologia. http://www.inmet.gov.br/portal/

Jank, L. (1995). Melhoramento e selecao de variedades de Panicum maximum. Proc 12 Th Simposio Sobre Manejo Da Pastagem, 1995, 21-58. http://ci.nii.ac.jp/naid/10025418717/en/

Köppen, W. (1936). Das geographische system der klimate. In Handbuch der Klimatologie (Vol. 1, pp. 1-44).

Latawiec, A. E., Strassburg, B. B. N., Valentim, J. F., Ramos, F., \& Alves-Pinto, H. N. (2014). Intensification of cattle ranching production systems: Socioeconomic and environmental synergies and risks in Brazil. Animal, 8(8), 1255-1263. https://doi.org/10.1017/S1751731114001566 
Sanches, G. M., Magalhães, P. S. G., Luciano, A. C. S., Camargo, L. A., \& Franco, H. C. J. (2020). Comprehensive assessment of spatial soil variability related to topographic parameters in sugarcane fields. Geoderma, 362, 114012. https://doi.org/10.1016/j.geoderma.2019.114012

Macedo Neto, A. A. L., Farias, P. R. S., Matos, G. S. B., Silva, G. B., Santos, A. V. F., \& Anhê, B. B. (2020). Diagnosis and spatial variability of soil fertility and crop production in a teak area in eastern Pará State. CERNE, 26(1), 37-47. https://doi.org/10.1590/01047760202026012683

Magalhães, A., Heinrichs, R., Meirelles, G. C., Lira, M. V. da S., Rebonatti, M. D., Bonini, C. S. B., Soares Filho, C. V., \& Moreira, A. (2020). Recuperação de pastagem de Urochloa decumbens com sistemas de manejo e adubação fosfatada. Research, Society and Development, 9(7), e347974118. https://doi.org/10.33448/rsd-v9i7.4118

Mantovanelli, B. C., Silva, D. A. P., Campos, M. C. C., Gomes, R. P., Soares, M. D. R., \& Santos, L. A. C. (2015). Avaliação dos atributos do solo sob diferentes usos na região de Humaitá, Amazonas. Revista de Ciências Agrárias, 58(2), 122-130. https://doi.org/10.4322/rca.1822

Maraschin, L., Scaramuzza, J. F., \& Vieira, C. R. (2020). Incubação do calcário e as características químicas de solos com texturas diferentes. Nativa, 8(1), 43. https://doi.org/10.31413/nativa.v8i1.6908

Marques Júnior, J., Sanchez, R. B., Pereira, G. T., \& Corá, J. . (2000). Variabilidade espacial de propriedades químicas e físicas de latossolos em áreas de cerrado sob cultivo de café, em Patrocínio, MG. O Estado-Da-Arte Da Agricultura de Precisão No Brasil, $105-112$.

Martha Júnior, G. B., Vilela, L., \& Sousa, D. M. G. de. (2007). Cerrado.

Matias, S. S. R., Nóbrega, J. C. A., Nóbrega, R. S. A., Andrade, F. R., \& Baptistel, A. C. (2015). Variabilidade espacial de atributos químicos em Latossolo cultivado de modo convencional com soja no cerrado piauiense. Revista Agro@mbiente On-Line, 9(1), 137-148. https://doi.org/10.5327/z19828470201500012036

Mombach, M. A., Carneiro Pedreira, B., Santos, L., Cabral, L. S., Silva, C., Santos, A., Mayra, A., \& Silva, P. (2019). Fator de resposta produtiva de mombaça e marandu ao déficit hídrico. Nativa, 7(6), 807-812. https://doi.org/10.31413/nativa.v7i6.7592

Montanari, R., Lima, E. de S., Lovera, L. H., Godoy, L. T. R., Henrique, A. F., \& Dalchiavon, F. C. (2013). Correlación de la productividad de un forraje con las propiedades físicas de un Ultisol en Aquidauana. Revista Ceres, 60(1), 102-110. https://www.redalyc.org/articulo.oa?id=305226999015

Montanari, R., Zambianco, E. C., Corrêa, A. R., Pellin, D. M. P., Carvalho, M. de P., \& Dalchiavon, F. C. (2012). Atributos físicos de um Latossolo Vermelho correlacionados linear e espacialmente com a consorciação de guandu com milheto. Revista Ceres, 59(1), 125-135. https://doi.org/10.1590/S0034737X2012000100018

Nachtigall, G. R., Nogueirol, R. C., \& Alleoni, L. R. F. (2007). Forms of copper in vineyard soils as affected by pH and poultry litter application. Pesquisa Agropecuaria Brasileira, 42(3), 427-434. https://doi.org/10.1590/s0100-204x2007000300017

Negreiros Neto, J. V., Santos, A. C., Guarnieri, A., Souza, D. J. A. T., Daronch, D. J., Dotto, M. A., \& Araújo, A. S. (2014). Variabilidade espacial de atributos físico-químicos de um Latossolo Vermelho-Amarelo distrófico em sistema plantio direto. Semina:Ciencias Agrarias, 35(1), 193-204. https://doi.org/10.5433/1679-0359.2014v35n1p193

Neves, S. M. A. S., Motinho, M. C., Neves, R. J., \& Soares, E. R. C. (2011). Estimativa da perda de solo por erosão hídrica na bacia hidrográfica do rio Jauru/MT. Sociedade \& Natureza, 23(3), 423-433. https://doi.org/10.1590/s1982-45132011000300005

Oliveira, B. D. A., Campos, L. P., Matias, S. S. R., Da Silva, T. S., \& Gualberto, A. V. S. (2021). Spatiality of soil chemical attributes in a banana cultivation area in west Bahia. Revista Caatinga, 34(1), 177-188. https://doi.org/10.1590/1983-21252021v34n118rc

Palermo, G. C., D'Avignon, A. L. de A., \& Freitas, M. A. V. (2014). Reduction of emissions from Brazilian cattle raising and the generation of energy: Intensification and confinement potentials. Energy Policy, 68, 28-38. https://doi.org/10.1016/j.enpol.2014.01.041

Pariz, C. M., Carvalho, M. P. e, Chioderoli, C. A., Nakayama, F. T., Andreotti, M., \& Montanari, R. (2011). Spatial variability of forage yield and soil physical attributes of a Brachiaria decumbens pasture in the Brazilian Cerrado. Revista Brasileira de Zootecnia, 40(10), 2111-2120. https://doi.org/10.1590/S151635982011001000007

Phelps, L. N., \& Kaplan, J. O. (2017). Land use for animal production in global change studies: Defining and characterizing a framework. In Global Change Biology (Vol. 23, Issue 11, pp. 4457-4471). Blackwell Publishing Ltd. https://doi.org/10.1111/gcb.13732

Portz, A., Martins, C. A. C., Silva, G. L. S., \& Zonta, E. (2015). Crescimento de capim Mombaça com aplicação de calcário e gesso em um Argissolo sul fluminense (1). 18 .

R Core Team. (2020). R: The R Project for Statistical Computing. https://www.r-project.org/

Ribeiro, A. C., Guimarães, P. T. G., \& Alvarez, V. H. (1999). Recomendações para o uso de corretivos e fertilizantes em Minas Gerais - 5a Aproximação.

Rodrigues, C. F. S., Lima, F. J. C., \& Barbosa, F. T. (2017). Importance of using basic statistics adequately in clinical research. Brazilian Journal of Anesthesiology (English Edition), 67(6), 619-625. https://doi.org/10.1016/j.bjane.2017.01.011

Salman, A. K. D., Soares, J. P. G., \& Canesin, R. C. (2006). Métodos de amostragem para avaliação quantitativa de pastagens. EMBRAPA - Empresa Brasileira de Pesquisa Agropecuária, April, 1-6. https://doi.org/0103-9334

Carneiro, J. S. S., Santos, A. C. M., Fidelis, R. R., Silva Neto, S. P., Santos, A. C., \& Silva, R. R. (2016). Diagnóstico e manejo da variabilidade espacial da fertilidade do solo no cerrado do Piauí. Revista de Ciências Agroambientais. 14(2). https://doi.org/10.5327/rcaa.v14i2.1469

Santana, W. D., Santos, A. C., Reis, A. D. S., Tavares, R. D. C., \& Freitas, G. A. de. (2019). Variabilidade espacial de atributos químicos do solo em sistema de agropecuária familiar no Tocantins. Científica, 47(3), 344. https://doi.org/10.15361/1984-5529.2019v47n3p344-357 
Research, Society and Development, v. 10, n. 14, e430101422038, 2021

(CC BY 4.0) | ISSN 2525-3409 | DOI: http://dx.doi.org/10.33448/rsd-v10i14.22038

Santos, H. G., Jacomine, P. K. T., Anjos, L. H. C., Oliveira, V. A., Lumbreras, J. F., Coelho, M. R., Almeida, J. A., Cunha, T. J. F., \& Oliveira, J. B. (2018). Sistema brasileiro de classificação de solos.

Schlotzhaver, S. D., \& Littell, R. C. (1997). SAS: system for elementary statistical analysis (C. SAS (2nd ed.).

Silva, E. R. O., Barros, M. M., Pereira, M. G., Gomes, J. H. G., \& Soares, S. D. C. (2020). Effects of spatial variability of soil chemical parameters on tifton 85 grass yield. Revista Caatinga, 33(1), 236-245. https://doi.org/10.1590/1983-21252020v33n125rc

Silva Júnior, F. de A., Barreto, P. A. B., Oliveira, F. G. R. B. de O., \& Pinto Junior, J. A. (2012). Atributos químicos de argissolo submetido a diferentes usos no sul da Bahia. ENCICLOPÉDIA BIOSFERA, 8(15), 2012.

Silva, S. de A., Lima, J. S. de S., Xavier, A. C., \& Teixeira, M. M. (2010). Variabilidade espacial de atributos químicos de um Latossolo Vermelho-Amarelo cultivado em plantio direto. Revista Ciência Agronômica, 44(1), 16-23. https://doi.org/10.1590/s1806-66902013000100003

SOFTWARE, G. D. (2018). Geoestatistics for the environmental sciences. https://geostatistics.com/

Somavilla, A., Marques, A. C. R., Caner, L., Oliveira, L. B., Quadros, F. L. F., Chabbi, A., Tiecher, T., \& Santos, D. R. (2021). Phosphate fertilization and liming in a trial conducted over 21 years: A survey for greater forage production and Pampa pasture conservation. European Journal of Agronomy, 125, 126259. https://doi.org/10.1016/J.EJA.2021.126259

Souza, Z. M. de, Cerri, D. G. P., Colet, M. J., Rodrigues, L. H. A., Magalhães, P. S. G., \& Mandoni, R. J. A. (2010). Análise dos atributos do solo e da produtividade da cultura de cana-de-açúcar com o uso da geoestatística e árvore de decisão. Ciência Rural, 40(4), 840-847. https://doi.org/10.1590/S010384782010005000048

Teixeira, P. C., Donagemma, G. K., Fontana, A., \& Teixeira, W. G. (2017). Manual De Metodos Logico-Cuantitativos (E. B. de P. A. (EMBRAPA) (3rd ed.). http://www.cse.edu.uy/sites/www.cse.edu.uy/files/documentos/Liccom_Camejo_2011-07-28.pdf

Tripathi, R., Nayak, A. K., Shahid, M., Lal, B., Gautam, P., Raja, R., Mohanty, S., Kumar, A., Panda, B. B., \& Sahoo, R. N. (2015). Delineation of soil management zones for a rice cultivated area in eastern India using fuzzy clustering. CATENA, 133, 128-136. https://doi.org/10.1016/J.CATENA.2015.05.009

Vanshchenko, Y., Piovesan, R. P., Lima, M. R., \& Favaretto, N. (2007). Solos e vegetação dos picos Camacuã, Camapuã e Tucum. Scientia Agraria, 4, 411419 .

VIEIRA, S. R. (2000). Geoestatística em estudos de variabilidade espacial do solo.

Vogado, R. F., Souza, H. A. de, Matias, S. S. R., Gualberto, A. V. S., Cunha, J. R. da, \& Leite, L. F. C. (2020). Spatial variability of carbon and nitrogen stocks in integrated management systems and pasture in a cerrado region. Research, Society and Development, 9(11), e67291110220. https://doi.org/10.33448/rsd-v9i11.10220

Walkley, A., \& Black, I. A. (1934). An examination of the Degtjareff method for determining soil organic matter and a proposed modification of the chromic acid titration method. Soil Science, 37(1), 29-38.

Zucoloto, M., Soares, J., Lima, D. S., \& Coelho, R. I. (2011). Correlação e variabilidade espacial de atributos químicos do solo e produção de bananeira "Prata-Anã". Revista Brasileira de Fruticultura. https://doi.org/10.1590/S0100-29452011000500064 